\title{
An investigation into the effectiveness of sensory modulation in reducing seclusion within an acute mental health unit
}

Chris Lloyd PhD

Senior Research Fellow, Behavioural Basis of Health, Griffith University Gold Coast Campus, Queensland Australia

\section{Robert King PhD}

Professor, School of Psychology and Counselling, Queensland University of Technology, Kelvin Grove, Queensland Australia

Tawanda Machingura BSc(hons), MHP(hons)

Team Leader, Mental Health Recovery Service, Gold Coast University Hospital, Southport, Queensland Australia

Address for correspondence:

Dr Chris Lloyd

Room 7.46, Griffith Health, G40

Griffith University Gold Coast Campus

Southport Q 4222

Email: c.lloyd@griffith.edu.au

Phone: 0756788699 


\begin{abstract}
In this paper we report the findings from an evaluation of the introduction of sensory modulation (SM) in an acute mental health inpatient unit. It was expected that SM could be used to help settle patients experiencing high levels of disturbance and that as a result, there would be less need for use of more restrictive seclusion practices. The evaluation took place in a hospital in south-east Queensland, Australia. SM was introduced in one acute unit while the other served as a control. The evaluation comprised two studies. In the first study we aimed to determine whether SM reduced the level of disturbance among patients given the opportunity to use it. In the second study we aimed to find out whether the introduction of SM reduced the frequency and duration of seclusion. In study 1, we found that most patients reported marked reduction in disturbance after using SM and there was a very large effect size for the group as a whole. In study 2 , we found that frequency of seclusion dropped dramatically in the unit that introduced SM but rose slightly in the unit that did not have access to SM. The change in seclusion rate was highly significant (chi square = 49.1, $d f=1, p<.001)$. Results are discussed, having reference to the limitations inherent in a naturalistic study.
\end{abstract}

Key words: sensory modulation, acute mental health, seclusion Word count: 4563 Type of paper: Research 


\section{Introduction}

Acute psychiatric inpatient units provide treatment for people who are experiencing symptoms that cannot be treated effectively in community settings. Symptoms such as delusions, hallucinations and elevated mood are common features of acute episodes of schizophrenia and bipolar disorder, which are the conditions most commonly treated in acute psychiatric units (Cleary, Hunt, \& Walter, 2010; Healy et al., 2012). Patients experiencing such symptoms often manifest behavioural disturbance, which presents management challenges for nurses who form the primary professional group staffing acute inpatient units (Bowers et al., 2009; Roberts, Crompton, Milligan \& Groves, 2009). A study conducted by Zhangi, Harvey, \& Andrew (2011) in Victoria, Australia found that the primary reason for admission was potential risk issues in $84 \%$ of admissions including a suicidal or self harm attempts and physical aggression towards humans or property. The most common diagnosis was psychosis and substance abuse. It was suggested that public mental health services mainly provide services to people with severe mental illness and a psychiatric admission is often preceded by behavioural disturbances that could not be managed in the community.

Aggression and violence are common occurrences during inpatient psychiatric admissions (Cleary et al., 2010). Strategies commonly used to manage behavioural disturbance include PRN medication and seclusion (isolation of the patient in locked room). A study conducted by Roberts et al. (2009) found that in a Queensland acute psychiatric inpatient unit, the local facility rate of seclusion was $12 \%$ of all patients admitted compared to a national average of $10 \%$. The re-seclusion rate of $76 \%$ was significantly higher than the national average of $31 \%$, and $87 \%$ of seclusion episodes were longer than 4 hours, compared with a national average of $41 \%$.

Both PRN medication and especially seclusion are viewed as last resort responses to disturbed behaviour and only to be used when interpersonal support and counselling have failed. Happell and Koehn (2010a) stated that seclusion is a coercive practice that has negative consequences for the well-being of patients. Recent Government policy has identified the reduction 
and, if possible, elimination of seclusion as a national safety priority (Happell \& Koehn, 2010b;

Happell \& Harrow, 2010). This reflects the principle that people experiencing mental illness should be treated in the least restrictive environment (Happell \& Koehn, 2011).

Queensland Health which has the responsibility for most acute psychiatric inpatient units in Queensland, Australia has been actively investigating less restrictive responses to behavioural disturbance in acute psychiatric inpatient units. One of these less restrictive responses is the use of sensory modulation (SM). S M, which is described in greater detail below, involves providing patients with access to sensory (mostly auditory and visual) stimulation designed to soothe and relax. In 2011, following recommendations by the Queensland Health Seclusion and Restraint Committee, S M was introduced into acute inpatient units at a limited number of sites. A training programme was conducted across the state to introduce S M to clinicians.

\section{Sensory Modulation}

SM is the neurological regulation of response to sensory stimuli (Kinnealey et al., 2011). Problems with sensory processing can result from difficulties in how the nervous system receives, organises, and uses sensory information from the body and the physical environment. This is necessary for self regulation, motor planning and skill development. For example auditory hallucinations may impact on self-concept, emotional regulation, attention, problem solving, behaviour control, skill performance, and the capacity to develop and maintain interpersonal relationships. In adults they may negatively impact on the ability to parent, work or engage in home management, social and leisure activities (AOTA, 2011; Brown \& Nicholson, 2011).

Decreased cognitive functioning, distortions in sensory processing, and the experience of psychological symptoms resulting from trauma and mental illness, can interfere with occupational engagement and often have a negative impact on sense of self and occupational performance skills (Brown \& Nicholson, 2011; Champagne 2011). Sensory processing refers to an individual's ability to 
take in information through the senses (touch, movement/balance, smell, taste, vision) and make a meaningful response (Brown \& Nicholson, 2011). This includes sensory modulation, sensory discrimination and sensory-based motor control, co-ordination and praxis (Champagne 2011). Interventions include activities or modalities offering specific types and amounts of sensation, at strategic times of use, for therapeutic purposes. For example this could involve having the patient sit in a rocking chair and listen to guided imagery CDs.

Champagne and Stromberg (2004) examined the sensory approaches used in an US inpatient psychiatric setting as an alternative to seclusion and restraint. A quality improvement approach was used to evaluate consumer perceptions of the effectiveness of sensory modulation rooms and sensory based approaches. Consumers rated their perceived levels of distress before and after each session using a qualitative questionnaire and a 10 -point ordinal rating scale. It was found that $89 \%$ of the sensory room sessions had a positive effect, $10 \%$ had no effect, and $1 \%$ had a negative effect. Patients experiencing the highest levels of distress at the beginning of each session reported the most substantial positive changes in perceived levels of distress after the use of the room. The facility's rates of seclusion and restraint were found to have decreased by $54 \%$ during the course of the year of implementation. Sivak (2012) found that following the introduction of comfort rooms (similar to SM) in one small rural tertiary mental health facility, positive outcomes were noted with decreased rates of client-to-client and client-to-staff assaults as well as zero use of seclusion and mechanical restraints.

An Australian study was conducted by Chalmers et al. (2012) who analysed data regarding sensory room use and acute arousal ratings within a high-dependency mental health unit. Preliminary data showed a significant reduction in patient distress levels, as measured using consumer and clinician ratings. A reduction was also found for acute arousal ratings, pre to post for the high dependency unit engagement programme. Chalmers et al. (2012) recommended cultural change and not just an environmental change, so as to build staff and consumers' confidence to 
utilise a variety of sensory based methods during times of need. Another Australian study found that the sensory room was an effective intervention to ameliorate distress and disturbed behaviour although this did not translate into reductions in overall rates of seclusion or aggression (Novak et al., 2012).

\section{Study Aims and Hypotheses}

The study had two aims. The first was to determine whether or not use of a sensory modulation environment reduced the level of distress experienced by patients in an acute psychiatric unit. The second was to determine whether availability of a sensory modulation room would reduce the use of seclusion as a response to patient disturbance. Two hypotheses informed the study:

1. It was hypothesised patients would report reduced distress after use of the SM environment.

2. It was hypothesised that the unit in which SM was introduced would have reduced frequency and duration of seclusion for the period after the introduction of SM compared with the period prior to the introduction of SM whereas the twin ward where SM was not available would show no such change.

\section{Method}

Study design This was a naturalistic study that was conducted in the acute mental health inpatient unit of a major Queensland hospital. One ward was used as the study group and the other ward served as the control group. This was not a standard experimental control as patients were not randomised to ward but rather were allocated on the basis of available beds. There were two interrelated studies. The first study examined the impact of the sensory modulation room on patient distress. This study used a repeated measures design. The second study examined changes in seclusion rates for two acute inpatient units, one of which offered a sensory modulation room and the other of which did not. This second study followed a prospective quasi experimental design 
whereby rates of seclusion in two acute wards were compared over a 12 month period (6 months before and 6 months after SM was introduced in one of the wards).

\section{Ethics Approval}

Approval for this study was granted by the hospital's Ethics Committee. Informed consent was not required for this study as it made use of data routinely collected for quality assurance purposes.

\section{Context}

This study was conducted in Acute Adult Mental Health Unit 1 (AAMH 1) also known as P1 and Acute Adult Mental Health Unit 2 (AAMH2) also known as P2 .P1 and P2 are both 20 bed facilities consisting of fourteen (14) Open Acute Care Beds and six (6) Psychiatric Intensive Care Unit (PICU) beds. During the course of the study, P1 introduced a sensory room for purposes of SM. A room consisting of two PICU beds were converted into one sensory room. Aside from this innovation, the two units were for all practical purposes interchangeable. They were both located within the same building and patients were admitted into one or other unit according to bed availability. The only systemic difference was that each had dedicated care teams, meaning that, while patients were probably equivalent there could have been differences in staff culture and clinical approach.

\section{Sample}

For study 1 , the sample consisted of all patients who used the sensory modulation room during a period of 6 months and who completed the pre and post rating scale (see below). For study two, the sample consisted of patients admitted to either P1 or P2 during the 12 month period from 1 January 2011 to 31 December 2011 and who were subject to seclusion. Altogether, 337 episodes of seclusion were documented during this period, 210 in P1 and 127 in P2. Of these episodes, 237 were of male patients and 99 were of female patients. We did not collect data on age or diagnosis 
as neither was central to the study. We did collect data on reasons for seclusion as recorded by the nurse who made the decision. Overwhelmingly the reason given was some form of aggression, more commonly verbal but sometimes physical. The term 'aggression' was most commonly used but other terms such as 'threatening', 'abusive' and 'hostile' were also used to describe what we took to be equivalent behaviour. In some instances nurses documented that the patient was 'agitated' without specifying aggression. Other documented descriptors were 'disorganised', 'demanding' and 'non-compliant'. Patients were very rarely secluded because of suicidality. P1 had a relatively greater proportion of seclusion episodes of female patients (36\%) compared with P2 (19\%) during this period (chi square $=10.5, \mathrm{df}=1, \mathrm{p}<.01$ ). While the chi square coefficient suggests this was probably not due to chance, we do not have data that would explain the reason for this difference. We think it is possible that it is attributable to the lower threshold for seclusion in P1. P2 may have been more willing to tolerate acting out by female patients on the grounds that it was less likely to dangerous.

\section{Intervention}

The SM room was designed in accordance with specifications described by Champagne \& Stromberg (2004). The components are set out in Table 1. Use of the SM room could be either patient initiated or staff initiated. Both staff and patients were provided with education and exposure to the SM room to assist them to make informed decisions about use of the facility. In general, both patients and staff were encouraged to use it for early intervention when one or other became aware of increasing patient distress.

Insert table 1 approximately here

Prior to the implementation of SM training was required. A training package comprising 3 distinct phases was developed. Phase 1: A SM Open Day was held where patients and staff were introduced to SM and had the opportunity to experience some of the modalities used in this 
approach. Phase 2: Basic SM training was provided to ward staff over a number of weeks. Phase 3: Ongoing one to one coaching was made available to staff and they can book themselves in with trainer

The introduction of SM Rooms required equipment. Funding was gained to implement the SM program. Five thousand dollars in total was allocated to fund equipment. The range of SM equipment purchased was purposefully selected to provide stimuli to the various sensory systems and address the individual and varied needs of consumers (see Table 1). As soon as practicable after admission, all patients undertook a sensory screen designed to identify the kinds of sensory stimulation likely to be calming. This screen informed modifications to the SM environment made for each individual who used it.

Initial access to the SM room was initiated by staff. The sensory stimulation made available to users was based on a standard screen applied to all patients that was designed to determine the kind of sensory stimulation most likely to be calming for the particular person. Once patients had used the room and found it helpful, they could request subsequent use of the room when feeling distressed. Staff could also recommend subsequent use of the room.

\section{Instruments and Data collection}

The Emotions Activity Rating Scale (Champagne \& Stromberg 2004) was the tool used to evaluate the impact of sensory modulation. This is a two item scale that asks the user to rate emotional state on a 10 point scale ranging from calm (1) to intense (10). The first item asks for retrospective rating of emotional state prior to use of the room and the second item asks for rating at present. The scale was administered when the patient left the sensory modulation room. This measure was chosen because it was developed to evaluate the impact of SM and its use enabled us to compare our findings with those of Champagne and Stromberg (2004). Seclusion rates and duration of seclusion were derived from records kept in a seclusion register and patient charts 


\section{Procedure}

For the first study, patients who used the SM room as an alternative to seclusion rated their distress using the instrument described above. For the second study, an audit of all seclusions during the calendar year 2011 was conducted. The SM room was introduced to P1 at the beginning of July 2011 and we compared seclusion rate for both P1 and P2 for the 6 month period prior to introduction of SM in P1 and for the 6 month period after the introduction of SM in P1.

\section{Results}

\section{Study 1: Impact of SM on self-rated distress}

Repeated measures analysis of scores on the Emotions Rating Scale showed a significant decline in severity of emotional distress after use of the SM room compared with prior to the use of the $\mathrm{SM}$ room $(F=79.3, \mathrm{df}=1 . \mathrm{P}<.001)$. Mean scores fell from 6.58 to 3.72 (mean decline $2.68,95 \%$ confidence interval 2.22 to 3.50 ) and the within subjects effect size was very large (partial eta squared $=.54)$. Person by person changes are presented in Figure 1 below.

Insert figure 1 approximately here

\section{Study 2 Impact of SM on frequency and duration of seclusion}

Table 2, below shows the number of seclusion episodes for each unit before and after sensory modulation was introduced into P1.

Insert table 2 approximately here

Table 2 shows that prior to 1 July 2011, when SM was introduced to P1, there were almost 4 times as many seclusion episodes in P1 as there were in P2. After SM was introduced, the rate of seclusion in P1 substantially decreased, whereas there was an increase in the rate of seclusion in P2. The rate of seclusion in P2, for the second half of the year, was substantially higher than it was in P1. The change in rate was highly significant (chi square $=49.1, \mathrm{df}=1, \mathrm{p}<.001$ ), 
While the rate of seclusion decreased significantly, there was no evidence that duration of seclusion episodes changed. Analysis of variance showed no main effect for either unit or period (prior to or after SM was introduced to P1) and no interaction between unit and period. The effect size (partial eta squared) was .24 with a 95\% confidence internal of .275 . This means that any effect of SM was on decision to use seclusion and not on the amount of time a person was secluded once a decision was made to use seclusion.

\section{Discussion}

We hypothesised in study 1 that patients using the SM environment would report reduced subjective distress. Consistent with the work of Chalmers and colleagues (2012), our data show that most patients who used SM did in fact have reduction in distress as measured by pre and post SM activity ratings. Typically the reduction was substantial and the overall effect size was very large. This means that the first hypothesis informing this study was supported.

We hypothesised in Study 2 that the frequency and duration of seclusion would reduce after the introduction of SM in acute mental health unit, P1. There was in fact a very substantial reduction in the frequency of seclusion following the introduction of SM to P1. Importantly there was no reduction but rather a small increase in the frequency of seclusion in the mirror unit, P2. However, there was no evidence that SM reduced the duration of seclusion, when it was used. While the total duration of seclusion fell dramatically in P1, this was entirely due to reduction in frequency of use of. The average duration of each seclusion episode when seclusion was used remained unchanged. This means that hypothesis 2 is partly supported.

The findings reported here provide preliminary evidence of the effectiveness of SM as an alternative to seclusion in an acute psychiatric inpatient environment. They suggest that SM has a positive impact on both patient self-reported well-being and on the frequency of use of seclusion, which is a restrictive practice. The findings from study 1 and from study 2, taken together, inform 
our understanding of how SM can work in an acute inpatient environment. As study 1 shows, SM has a soothing effect on disturbed patients. As our findings from study 2 show, because of this soothing effect, nurses do not need recourse to the more restrictive practice of seclusion nearly as frequently.

The findings must be considered in the light of the limitations set out below but the size of the observed impact suggests that, notwithstanding these limitations, SM is likely to yield real benefits both for patients and for staff. High levels of subjective distress are to be expected in an acute inpatient environment and, in the absence of an effective therapeutic response, can lead to escalation and risk of harm to patients and staff. The evidence from this study suggests that SM can form a valuable component of an effective therapeutic response to patient distress. These conclusions are consistent with the findings of Novak et al. (2012) and Sivak (2012).

While there was a clear and very substantial reduction in the frequency of seclusion in P1 after the introduction of SM, there was no reduction in the duration of the reduced number of seclusion episodes that did occur. We think this is probably because those patients who were unwilling to use SM or who were judged by nurses to be unsuitable for SM were very disturbed and needed time to settle. The fact that there was no increase in duration of seclusion is in fact a positive indicator as the secluded group in the period after the introduction of SM were almost certainly a more disturbed group of patients than the mark larger group secluded during the period prior to the introduction of SM.

\section{Limitations}

There are limitations that require caution in interpretation of findings in both study 1 and study 2 . In study 1 , the scale used was specifically designed for evaluation of SM and has been used in previous studies but its psychometric properties have not been adequately investigated. Retrospective rating of level of distress prior to use of SM may also introduce error. However, some 
users of the SM room would not have been able to complete even a simple measure prior to use of the room and we considered it better to use a standard administration point rather than have some patients completing the scale prior to SM and some after. The age and diagnosis of the participants may be potential confounders.

The most important limitation of study 2 is that the naturalistic design of the study means that caution is required in attribution of causality. Patients were not randomly assigned to P1 or P2 and there was no established control group. However it is also the case that the only factor that determined whether a patient was admitted to $\mathrm{P} 1$ or $\mathrm{P} 2$ was bed availability and there is no reason to suspect that patients admitted to P1 after the introduction of SM had different risk of seclusion than those admitted to P2. We therefore think the absence of random assignment is unlikely to have had a major bearing on results. A more serious limitation of the naturalistic design is that it was not possible to control possible confounds. The introduction of SM to P1 and the formal evaluation of SM meant that nursing staff who made decisions about seclusion were not blind to the purposes of SM and may well have felt they were under scrutiny. Queensland Health policy clearly favoured reduction of frequency of use of seclusion and it is possible that the introduction of SM and the evaluation of SM impact influenced decision-making about seclusion

\section{Treatment implications and recommendations}

1. The findings from study 1 show that use of SM does substantially reduce the level of patient disturbance.

2. The dramatic turnaround in seclusion rate after the introduction of SM suggests that the availability of SM can reduce the frequency of use of seclusion as a response to patient disturbance in an acute inpatient unit.

3. There is need for further research that is designed so as to better address the limitations we have noted above. In particular, we think that better measures are required - both patient 
rating measures and observer rated measures. We also think that studies that investigate the longer-term impact of SM are warranted.

\section{Conclusion}

The successful implementation of SM requires certain key ingredients; training of staff, equipment, a multidisciplinary approach and ongoing marketing. Findings indicate that sensory approaches do impact positively on self rated distress and frequency of seclusion episodes. Further research is needed to explore the long-term effectiveness of SM in acute inpatient units and the application of this approach in other mental health settings.

\section{Acknowledgements}

A special thank you to Chris Foley for all his hard work managing the data base and Maria Shepherd for assistance with data collection and entry, and to the sensory modulation working group members. 


\section{References}

AOTA (2011). Fact sheet. Occupational therapy using a sensory integration-based approach with adult populations. Bethesda, MD: American Occupational Therapy Association, Inc.

Bowers, L., Allan, T., Simpson, A., Jones, J., van der Merwe, M., \& Jeffery, D. (2009). Identifying key factors associated with aggression on acute inpatient psychiatric wards. Issues in Mental Health Nursing, 30, 260-271.

Brown, C., \& Nicholson, R. Sensory skills. In: C. Brown \& V.O. Stoffel, Occupational therapy in mental health - a vision for participation. Philadelphia: FA Davis Company, 280-297.

Chalmers A, Harrison S, Mollison K, Molloy N, Gray K (2012). Establishing sensory-based approaches in mental health inpatient care: A multidisciplinary approach. Australasian Psychiatry, 20(1), 35-39.

Champagne T (2011). The influence of posttraumatic stress disorder, depression, and sensory processing patterns on occupational engagement: A case study. Work, 38(1), 67-75.

Champagne T, Stromberg N (2004). Sensory approaches in inpatient psychiatric settings. Innovative alternatives to seclusion and restraint. Journal of Psychosocial Nursing, 43(9), 35-44.

Cleary, M., Hunt, G.E., \& Walter, G. (2010). Seclusion and its context in acute inpatient psychiatric care. Journal of Medical Ethics, 36, 459-462.

Healy, D., Le Noury, J., Linden, S.C., Harris, M., Whitaker, C., Linden. D., Baker, D., \& Roberts, A.P. (2012). The incidence of admissions for schizophrenia and related psychoses in two cohorts: 
1875-1924 and 1994-2010. BMJ Open 2012;2:e000447.doi:10.1136/bmjopen-2011-000447

Happell, B., \& Harrow, A. (2010). Nurses' attitudes to the use of seclusion: A review of the literature. International Journal of Mental Health Nursing, 19, 162-168.

Happell, B., \& Koehn, S. (2010a). From numbers to understanding: The impact of demographic factors on seclusion rates. International Journal of Mental Health Nursing, 19, 169-176.

Happell, B., \& Koehn, S. (2010b). Attitudes to the use of seclusion: has contemporary mental health policy made a difference? Journal of Clinical Nursing, 19, 3208-3217.

Happell, B., \& Koehn, S. (2011). Impacts of seclusion and the seclusion room: Exploring the perceptions of mental health nurses in Australia. Archives of Psychiatric Nursing, 25, 109119.

Kinnealey, M., Koenig, K.P., \& Smith, S. (2011). Relationships between sensory modulation and social supports and health-related quality of life. American Journal of Occupational Therapy, 65, 320-327.

Novak, R., Scanlan, J., McCaul, D., MacDonald, N., \& Clarke, T. (2012).Pilot study of a sensory room in an acute inpatient psychiatric unit. Australasian Psychiatry, 20, 401-406.

Roberts, D., Crompton, D., Milligan, E., \& Groves, A. (2009). Reflections on the use of seclusion in an acute mental health facility. Journal of Psychosocial Nursing, 47, 25-31.

Sivak, K. (2012). Implementation of comfort rooms to reduce seclusion, restraint use and acting out behaviours. Journal of Psychosocial Nursing, 50, 24-34. 
Zhangi, J., Harvey, C., \& Andrew, c. (2011). Factors associated with length of stay and the risk of readmission in an acute psychiatric inpatient facility: a retrospective study.

Australian and New Zealand Journal of Psychiatry, 45, 578-585.

Table 1 The different sensory systems and examples of items or interventions

\begin{tabular}{|c|c|}
\hline $\begin{array}{l}\text { Proprioceptive } \\
\text { - Resistive had-squeeze equipment } \\
\text { - Use of therapy ball } \\
\text { - Use of weighted ball activities } \\
\text { - Dance/yoga/tai chi }\end{array}$ & $\begin{array}{l}\text { Vestibular } \\
\begin{array}{l}\text { - } \text { Rocking chair } \\
\text { - Glider rocker } \\
\text { - Therapy ball activities } \\
\text { - Swinging } \\
\text { - } \text { Detting in and out of beanbag chair }\end{array}\end{array}$ \\
\hline 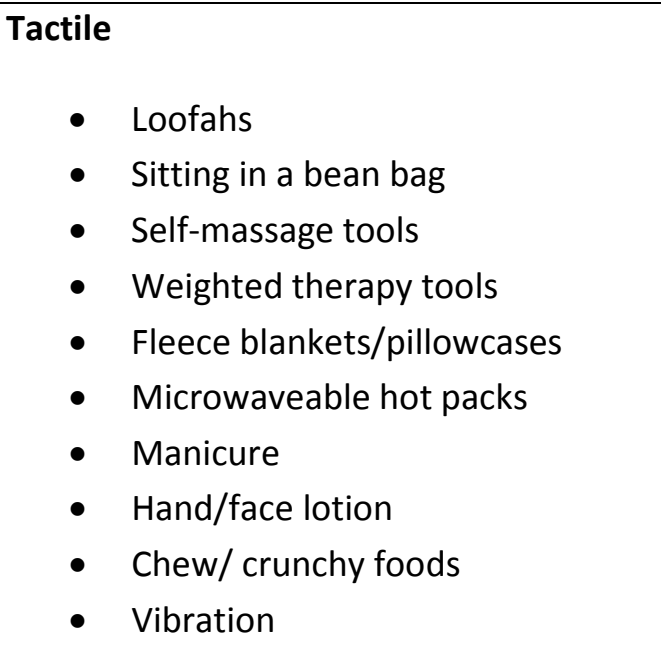 & $\begin{array}{l}\text { Auditory } \\
\begin{array}{l}\text { - } \text { Guided imagery CDs } \\
\text { - } \text { Various types of music - fast/slow pace } \\
\text { - } \text { Stereo or personal audio system } \\
\text { - } \text { Musical instruments }\end{array}\end{array}$ \\
\hline $\begin{array}{ll}\text { Visual } & \\
\text { - } & \text { Bubble lamp } \\
\text { - } & \text { Wall murals/various pictures } \\
\text { - Bubbles } \\
\text { - Glitter wands } \\
\text { - Various lights/lighting } \\
\text { - Computer use } \\
\text { - Coloured sunglasses }\end{array}$ & $\begin{array}{l}\text { Olfaction } \\
\begin{array}{l}\text { - Scented bath/body wash } \\
\text { - Scented lotions/balms } \\
\text { - Aromatherapy ( } 100 \% \text { essential oils) } \\
\text { - Lemon } \\
\text { - Eucalyptus } \\
\text { - Candles } \\
\text { - Herbal teas }\end{array}\end{array}$ \\
\hline $\begin{array}{l}\text { Gustatory } \\
\text { - Carrot sticks }\end{array}$ & \\
\hline
\end{tabular}


- Celery sticks

- Pretzels

- Sour lollies

- Gummy bears

- Fruit snacks

- Ice blocks/frozen fruit bars

- Ice

- Biting on a lemon

- Chewy foods

- Strong mints

- Herbal teas

- Lollipops

- Trail mix

- Yoghurt 
Figure 1. Impact of sensory modulation on self-rated distress

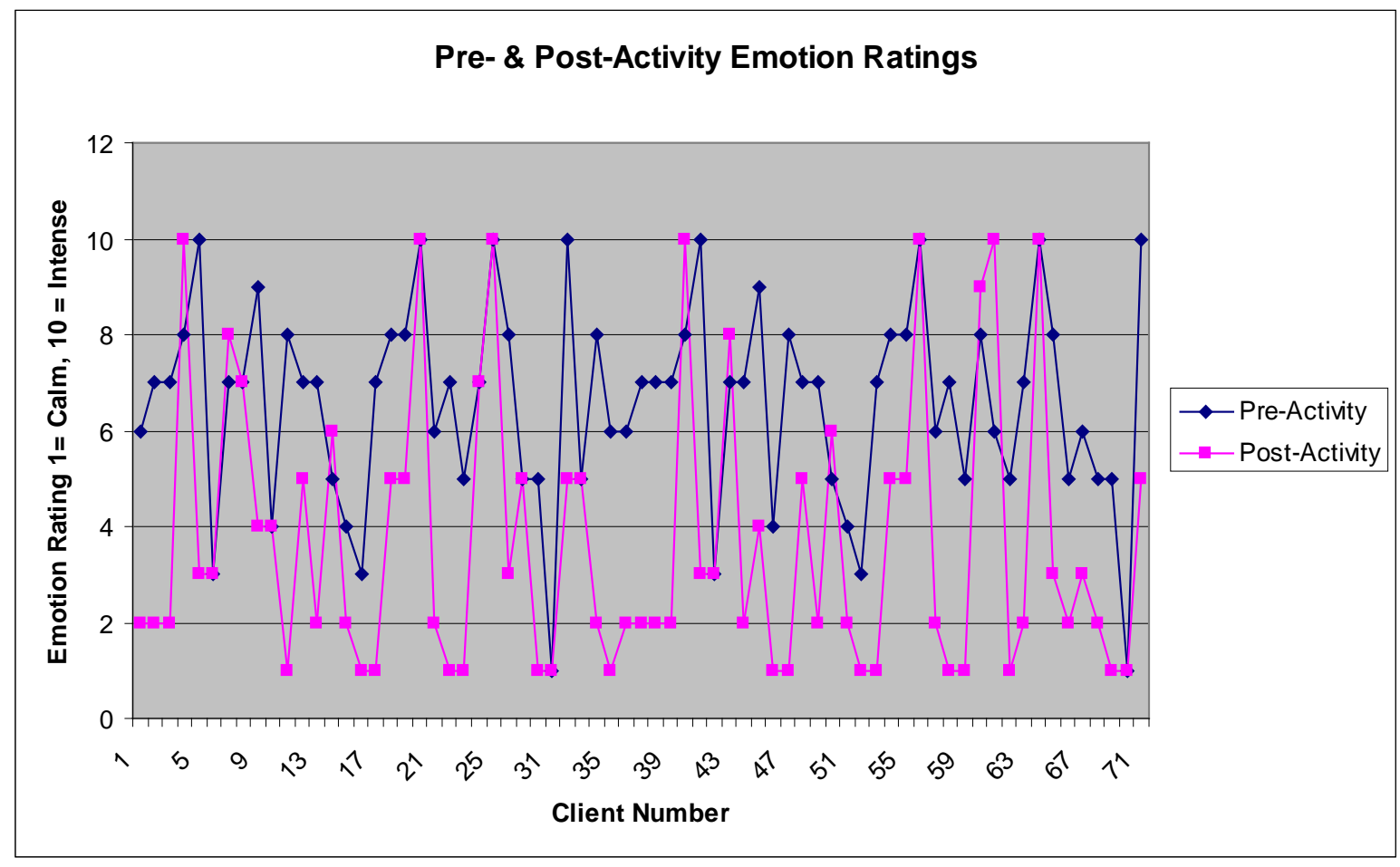


Table 2. Impact of sensory modulation on seclusion

\begin{tabular}{|l|l|l|}
\hline & Seclusion episodes in P1 & Seclusion episodes in P2 \\
\hline $\begin{array}{l}\text { Prior to introduction of SM to } \\
\text { P1 }\end{array}$ & 157 & 46 \\
\hline After introduction of SM to P1 & 53 & 81 \\
\hline
\end{tabular}

